\title{
Effects of contextual fear conditioning and pentylenetetrazol on panic-like reactions induced by dorsal periaqueductal gray stimulation with N-methyl-D-aspartate
}

\author{
Bruno de Oliveira Galvão ${ }^{1}$, Bruno Costa Larrubia ${ }^{1}$, Wouter Jan Hommes ${ }^{2}$, Luis Fernando \\ Cardenas $^{3}$, Antonio Pedro de Mello Cruz ${ }^{4}$ and Jesus Landeira-Fernandez ${ }^{1,5}$ \\ 1 Pontifícia Universidade Católica do Rio de Janeiro, Brazil \\ 2 University of Maastricht, Netherlands \\ 3 Universidad de Los Andes, Colombia \\ 4 Universidade de Brasília, Brazil \\ 5 Universidade Estácio de Sá, Brazil
}

\begin{abstract}
Electrical or chemical stimulation of the dorsal periaqueductal gray (DPAG) has been accepted as an animal model of panic attacks. This study investigates the influence of anticipatory anxiety in the occurrence of panic-like behavior induced by N-methyl-Daspartate (NMDA) microinjection into the DPAG of rats. Behavioral (i.e., contextual fear conditioning) and pharmacological (i.e., pentylenetetrazol) manipulations were employed as animal models of anticipatory anxiety. In the first experiment, animals exposed to contextual cues that had been previously associated with electric footshocks through contextual fear conditioning were less likely than non-conditioned control animals to display defensive reactions such as running and jumping in response to microinjection of NMDA $(0.3 \mu \mathrm{l}$ of $15.0 \mu \mathrm{g} / \mu \mathrm{l})$ into the DPAG. In the second experiment, rats were injected intraperitoneally with the anxiogenic drug pentylenetetrazol (PTZ, $15 \mathrm{mg} / \mathrm{kg}$ ) 5 minutes before receiving intra-DPAG microinfusion with the same dose of NMDA as in Experiment 1. Panic-related behaviors were registered in an experimental arena immediately after NMDA microinfusion. As compared with saline pre-treated animals, PTZ significantly attenuated NMDA-induced panic-like reactions. These results further demonstrate the usefulness of DPAG chemical stimulation as an animal model of panic attacks and suggest that behavioral and pharmacological activation of the brain mechanisms underlying anticipatory anxiety might exert an antipanic-like effect. Keywords: anxiety disorders, contextual fear conditioning, defensive behavior, N-methyl-D-aspartate, panic attack, periaqueductal gray, pentylenetetrazole.
\end{abstract}

Received 2 February 2010; received in revised form 6 May 2010; accepted 10 May 2010. Available on line 26 June 2010

\section{Introduction}

Anxiety disorders constitute a heterogeneous group of related nosological categories. According to the DSMIV (1995), the diagnosis of generalized anxiety disorder (GAD) involves the presence of constant worry associated with several symptoms such as irritability, feeling tired and restless, and concentration difficulties. This condition can also lead to a variety of somatic symptoms including chronic

Bruno de Oliveira Galvão, Departamento de Psicologia, Pontifícia Universidade Católica do Rio de Janeiro, Brazil. Bruno Costa Larrubia, Departamento de Psicologia, Pontifícia Universidade Católica do Rio de Janeiro, Brazil. Wouter Jan Hommes, Faculty of Psychology, University of Maastricht, Netherlands. Luis Fernando Cardenas, Departamento de Psicologia, Laboratório de Neurociencia y Comportamiento Universidad de Los Andes, Bogotá, Colombia. Antonio Pedro de Mello Cruz, Instituto de Psicologia, Universidade de diarrhea, muscle pain, headache, palpitation, tachycardia and gastrointestinal dysfunction. GAD is different from panic attacks, which are characterized by sudden surges of thoughts that death is imminent, or loss of control accompanied by major neurovegetative changes such as palpitations, difficulty breathing deeply, sweating, shaking, increased peristalsis and hypertension. Panic attacks are among the most prominent symptoms in panic disorder (PD), but they can occur in other anxiety disorders.

Brasília, Brazil. Jesus Landeira-Fernandez, Departamento de Psicologia, Pontifícia Universidade Católica do Rio de Janeiro, Brazil and Curso de Psicologia, Universidade Estácio de Sá, Rio de Janeiro, Brazil. Correspondence regarding this article should be directed to: J Landeira-Fernandez, Departamento de Psicologia, Pontifícia Universidade Católica do Rio de Janeiro, PUC-Rio, Rua Marquês de São Vicente, 225, Gávea, Rio de Janeiro, RJ, Brazil, CEP 22453-900. Phone: +55-21-35271186. Fax:+55-21-3527-1187. E-mail:landeira@puc-rio.br 
Since the noteworthy work of Klein and Fink (1962), which showed that imipramine had a selective effect in the treatment of panic attacks, the relationship between GAD and panic attacks has been a subject of intense debate. The more traditional view suggests that anticipatory anxiety and panic attacks are part of the same continuum. According to this interpretation, an increase in anxiety may result in a panic attack episode (Bouton, Mineka, \& Barlow, 2001). In the same way, PD patients report that the majority of panic attack episodes occur when the patient is already at a plateau of extremely high anxiety (Basoglu, Marks, \& Sengun, 1992). Finally, patients who had panic attacks induced by sodium lactate infusion reported a high level of anxiety immediately before the occurrence of a panic attack event (Liebowitz et al. 1984).

The alternative view proposes that anxiety and panic attacks are qualitatively different pathologies and that they have a multifaceted relationship. According to this view, the occurrence of panic attacks can trigger intense anxiety reactions, but anxiety symptoms can inhibit the occurrence of panic attacks. Some clinical evidence supports this suggestion. For example, relaxation techniques employed during the treatment of anxiety symptoms can induce panic attacks (Adler, Craske, \& Barlow, 1987). Moreover, panic attacks seem to occur more frequently during the initial course of PD when anxiety levels are relatively low, compared to later PD stages when the anxiety symptoms are extremely high (Klein \& Klein, 1989). Finally, pharmacological results also corroborate the view that increasing anxiety might inhibit the occurrence of panic attacks. Patients diagnosed with PD and treated with serotonergic agonists presented a decreased occurrence of panic attacks (Cassano, Perugi, \& McNair, 1988; Gorman et al. 1987), but showed an increase in anxiety symptoms (Targum \& Marshall, 1989).

One possible explanation for these conflicting results is the fact that clinical expressions of different anxiety disorders are extremely complex (Kemp \& Felmingham, 2008). In fact, PD is commonly associated with agoraphobia as well as with other anxiety or affective disorders (Hettema, 2008). Therefore, experimental research employing animal models of anxiety disorders is an important avenue of research to improve our understanding of the relationship between GAD and panic attacks.

Contextual fear conditioning represents one of the most useful animal models of GAD (see Brandão et al., 2008 for a review). In a typical experiment, a rat is exposed to a novel chamber and a brief unsignaled footshock is presented several minutes later. Some time later (i.e. next day), the animal freezes when returned to the same chamber in the absence of the aversive stimulus (LandeiraFernandez, 1996). This conditioned freezing is weakened by anxiety-reducing drugs (Fanselow et al., 1991) and enhanced by anxiety-inducing drugs (Izumi et al., 1999), giving it predictive value for screening pharmacological substances that modulate general anxiety disorder in humans. Pharmacological results have also indicated that the running response induced by chemical or electrical stimulation of the dorsal portion of the periaqueductal gray matter (DPAG) can serve as an important model of panic attacks (see Brandão et al., 2008 for a review). Panicolytic drugs such as clomipramine and fluoxetine increased the threshold electrical current required to elicit running behaviors (Vargas \& Schenberg, 2001). In humans, DPAG electrical stimulation produced closely related panic attack symptoms such as heart-pounding terror and feelings of imminent death accompanied by diffuse face and chest pain (Nashold, Wilson, \& Slaughter, 1969).

Previous results from our laboratory indicated that rats exposed to contextual cues previously associated with electrical footshocks engaged in robust defensive freezing behavior and were less likely to display running behavior induced by electrical stimulation of the DPAG when compared with control animals that were not exposed to contextual fear conditioning (Magierek et al., 2003). These results corroborate the view that anxiety produced an inhibitory effect on panic-like behavior. The purpose of this study is to further investigate this issue. In the first experiment, the Magierek et al. (2003) study was replicated using chemical instead of electrical stimulation of the DPAG as a model of panic attack. The second experiment also investigated the influence of anxiety in the occurrence of panic attacks. As in the first experiment, DPAG chemical stimulation was used as a model of panic attack, whereas pentylenetetrazole (PTZ) instead of contextual fear conditioning was employed as the anxiety inducing procedure.

\section{Experiment 1: Effect of contextual fear conditioning on the escape responses induced by chemical stimulation of the DPAG}

\section{Method}

Subjects

Malealbino rats from the animal colony ofPUC-Rio's Psychology Department were used as subjects. Room temperature was controlled $\left(24 \pm 1^{\circ} \mathrm{C}\right)$ and the light-dark cycle was maintained on a $12 \mathrm{hr}$ on-off cycle. Animals were between 90 to 120 days old at the beginning of the experiment and were housed individually in plexiglass cages and given free access to food and water throughout the experiment. All experimental protocols employed in this work are in accordance with the Brazilian Society of Neuroscience and Behavior Guidelines for Care and Use of Laboratory Animals (SBNeC), which are based on the US National Institutes of Health Guide for Care and Use of Laboratory Animals (revised in 1996). 


\section{Apparatus}

The contextual fear conditioning took place in a wooden observational arena measuring 90 X 85 X 90 $\mathrm{cm}$. The floor of the arena was composed of 55 stainless steel rods with a diameter of $1 \mathrm{~mm}$ and spaced $1 \mathrm{~cm}$ apart (center to center). These rods were connected to a shock generator and scrambler (AVS, SCR04; São Paulo) with an interface that allowed the experimenter to apply electrical footshocks. A red light bulb (40 watts) in the ceiling of the experimental room and a small camera placed above the arena allowed the experimenter to observe the animal's behavior on a monitor located outside the experimental room.

\section{Surgery}

All animals were implanted with a unilateral guide cannula made of stainless steel aimed at the DPAG. Under tribromoethanol anesthesia $(250 \mathrm{mg} / \mathrm{kg}$, i.p.), each animal was fixed in a Kopf stereotaxic frame and injected locally with lidocaine $(20 \mathrm{mg} / \mathrm{ml})$. The upper incisor bar was set at $3.3 \mathrm{~mm}$ below the interaural line such that the skull was horizontal between bregma and lambda. The following coordinates were employed for implantation of the guide cannula, with the lambda serving as the reference for each plane according to the Paxinos and Watson (1986) rat brain atlas: anteroposterior $(\mathrm{AP})=+2.3 \mathrm{~mm}$; mediolateral $(\mathrm{ML})=$ $-1.7 \mathrm{~mm}$; and dorsoventral $(\mathrm{DV})=-4.5 \mathrm{~mm}$. The guide cannula was attached to the skull of the animal with acrylic resin and two steel screws. A stylet with a guide cannula of the same length was introduced inside the guide cannula to prevent obstruction.

\section{Intracerebral Injection}

The experiment began 1 week after surgery. NMDA microinjection (Sigma, St. Louis, USA) was performed using an internal cannula (22 ga, Plastic One) that was introduced $0.5 \mathrm{~mm}$ below the guide cannula. The injection cannula was connected to a $5 \mu 1$ Hamilton syringe via PE tubing and $0.3 \mu \mathrm{l}$ of NMDA $(15.0 \mu \mathrm{g} / \mu \mathrm{l})$ or saline was microinjected. The displacement of an air bubble inside the PE tubing was used to monitor NMDA microinjection.

\section{Procedure}

Animals in the first group (conditioning/NMDA) were placed in the observational arena for 5 minutes. At the end of this period, three unsignaled electrical footshocks $(1 \mathrm{~mA}, 1 \mathrm{sec})$ were delivered with a 20 second intershock interval. The animals were returned to their home cages 1 minute after the last shock. The testing session occurred 6 hours after the contextual fear conditioning training and lasted 30 minutes. Animals were microinjected with NMDA into the DPAG and immediately placed in the same arena where the three footshocks had been previously administered. Animals in the second group (conditioning/saline) underwent exactly the same procedure except that saline was microinjected into the DPAG before the beginning of the testing session instead of NMDA. Animals in the third group (no conditioning/NMDA) underwent the same procedure as the first group without any footshocks. Finally, animals in the fourth group (no conditioning/ saline) did not undergo the contextual fear conditioning procedure and saline was microinjected into the DPAG before testing. Running and jumping behaviors were scored during the test session. Running was defined as fast locomotion with elevation of the trunk and tail and swinging movements of contralateral limbs (Bittencourt et al, 2004). Jumping was scored when the animal presented four paws in the air.

\section{Histology}

At the end of the experiment, animals were deeply anaesthetized with chloral hydrate and perfused intracardially with saline solution $(0.9 \%)$ followed by formalin solution $(10 \%)$. The cannulas, as well as the brains, were removed and placed in a $10 \%$ formalin solution. Three days later, the brains were frozen and $50 \mu \mathrm{m}$ brain sections were cut using a microtome and stained with cresyl blue to localize cannula positions.

\section{Data analysis}

Previous experiments have shown that saline microinjection into the DPAG does not lead to aversive responses such as running and jumping (Molchanov \& Guimarães, 1999). This implies that no variance should occur in the groups that were microinjected with saline and that the between-group variance should differ according to treatment. Therefore, nonparametric statistics were used to analyze the present results. The Kruskal-Wallis test was used to determine overall differences among groups, followed by the MannWhitney test for pairwise comparison.

\section{Results}

Histological examination of the brain slices indicated that the internal cannula were located inside or at the borders of DPAG. Animals with cannula outside the DPAG were excluded from statistical analysis. The final sizes of each of the four groups were as follows: conditioning/NMDA, $\mathrm{N}=6$; conditioning/saline, $\mathrm{N}=6$; no conditioning/NMDA, $\mathrm{N}=6$; no conditioning/saline, $\mathrm{N}=7$.

Table 1 shows the median running and jumping frequency as well as running time following NMDA or saline microinjection into the DPAG among animals with or without contextual fear conditioning. A KruskalWallis test indicated a significant difference among groups in the occurrence of jumping frequency, $(\mathrm{H}(3)=$ $10.77 ; \mathrm{p}<.05)$. A post hoc analysis performed with the Mann Whitney test indicated that the no conditioning/ 
Table 1 - Median (minimum - maximum) of running and jumping behaviors in rats treated with DPAG microinjection of $\operatorname{NMDA}(.3 \mu \mathrm{l}$ of $15.0 \mu \mathrm{g} / \mu \mathrm{l})$ or saline among animals exposed (presence) or not exposed (absence) to contextual cues previously associated with footshock (contextual fear conditioning).

\begin{tabular}{llll}
\hline \multirow{2}{*}{ Behavioral Output } & Contextual Fear Conditioning & \multicolumn{2}{l}{ DPAG Microinjection } \\
\cline { 3 - 4 } & & Saline & NMDA \\
\hline Jumping frequency & Absent & $0(0-0)$ & $3.4(0-25)$ \\
& Present & $0(0-0)$ & $0(0-7)$ \\
Running frequency & Absent & $0(0-0)$ & $0(0-0)$ \\
& Present & $0(0-0)$ & $9.5(0-68)$ \\
Runing time (sec) & Absent & $0(0-0)$ & $0(0-0)$ \\
\hline
\end{tabular}

NMDA group presented no significant difference in jumping behavior when compared to the conditioning/ NMDA group $(p>.05)$ and significantly higher jumping behavior when compared to other two groups (ps < .05). A Kruskal-Wallis test also indicated a significant difference among groups in the occurrence of running frequency, $(\mathrm{H}(3)=15.0 ; \mathrm{p}<.05)$. A post hoc analysis performed with the Mann Whitney test indicated that the no conditioning/NMDA group presented a significantly higher running frequency when compared to the other three groups ( $\mathrm{ps}<.05$ for all comparisons). Finally, a Kruskal-Wallis test indicated a significant difference among groups in relation to running time $(\mathrm{H}(3)=15.0$; $\mathrm{p}<.01$.). A post hoc analysis performed with the Mann Whitney test indicated that the no conditioning/NMDA group had a significantly higher different running time when compared to the others three groups ( $\mathrm{ps}<.05$ for all comparisons).

\section{Experiment 2: Effect of pentylenetetrazol on the escape responses induced by chemical stimulation of the DPAG}

\section{Methods}

Animals and apparatus

This experiment used rats with the same characteristics as described in experiment 1 , and the experiment took place in the same arena.

\section{Procedure}

All the animals were submitted to the same surgical procedure described in Experiment 1 for implanting the unilateral guide cannula into the DPAG. One week after surgery, the first group of animals (PTZ/NMDA) received an i.p. injection of PTZ $(15 \mathrm{mg} / \mathrm{ml}$; SigmaAldrich, Poland) five minutes before being introduced to the observational arena. The PTZ dose employed in this study was chosen based on previous work from our group (Cruz, Frei, \& Graeff, 1994) as well as from reports in the literature (Ramos et al., 2008). Immediately before being placed into the observational arena, each animal received an NMDA $(0.3 \mu \mathrm{l} / 15.0 \mu \mathrm{g} / \mu \mathrm{l})$ microinjection into the DPAG. Animals in the second group (Saline/ NMDA) underwent exactly the same procedure as the first group with the exception that saline $(0.9 \%)$ instead of PTZ was injected intraperitoneally. As in the first experiment, running and jumping behavior were scored during a 30 minute test session.

\section{Results}

Histological examination of brain slices indicated that internal cannulas were located within the DPAG area. Animals with cannulas outside the DPAG were excluded from statistical analysis. The final group samples were: Saline/NMDA, N=14; PTZ/NMDA, N=12.

Figure 1 presents the mean $( \pm \mathrm{SEM})$ of jumping frequency (upper portion), running frequency (middle portion) and running time (lower portion). The student's t-test was employed to evaluate statistical differences between groups for these three behavioral measurements. The analyses indicated a reliable difference in jumping frequency $\left(\mathrm{t}_{(24)^{\circ}}=2.8 ; \mathrm{p}<.001\right)$, running frequency $\left(\mathrm{t}_{(24)}\right.$ $=3.7 ; \mathrm{p}<.001)$ and running time $\left(\mathrm{t}_{(24)}=4.5 ; \mathrm{p}<.001\right)$. All behavioral indices indicated that escape responses induced by NMDA microinjections into the DPAG were lower among animals injected with PTZ when compared to control groups that received i.p. injections of saline.

\section{Discussion}

Chemical stimulation of DPAG resulted in welldefined escape/defensive behaviors such as running and jumping. This behavioral outcome has also been reported by other studies (Bandler \& Carrive, 1988; Behbehani, 1995; Krieger \& Graeff, 1985). The first experiment indicated that contextual fear conditioning was capable of inhibiting escape behavior induced by NMDA microinjection into the DPAG. These results extend previous findings reported by Magierek et al. (2003) and are in agreement with the view that anticipatory anxiety (as modeled in this experiment 


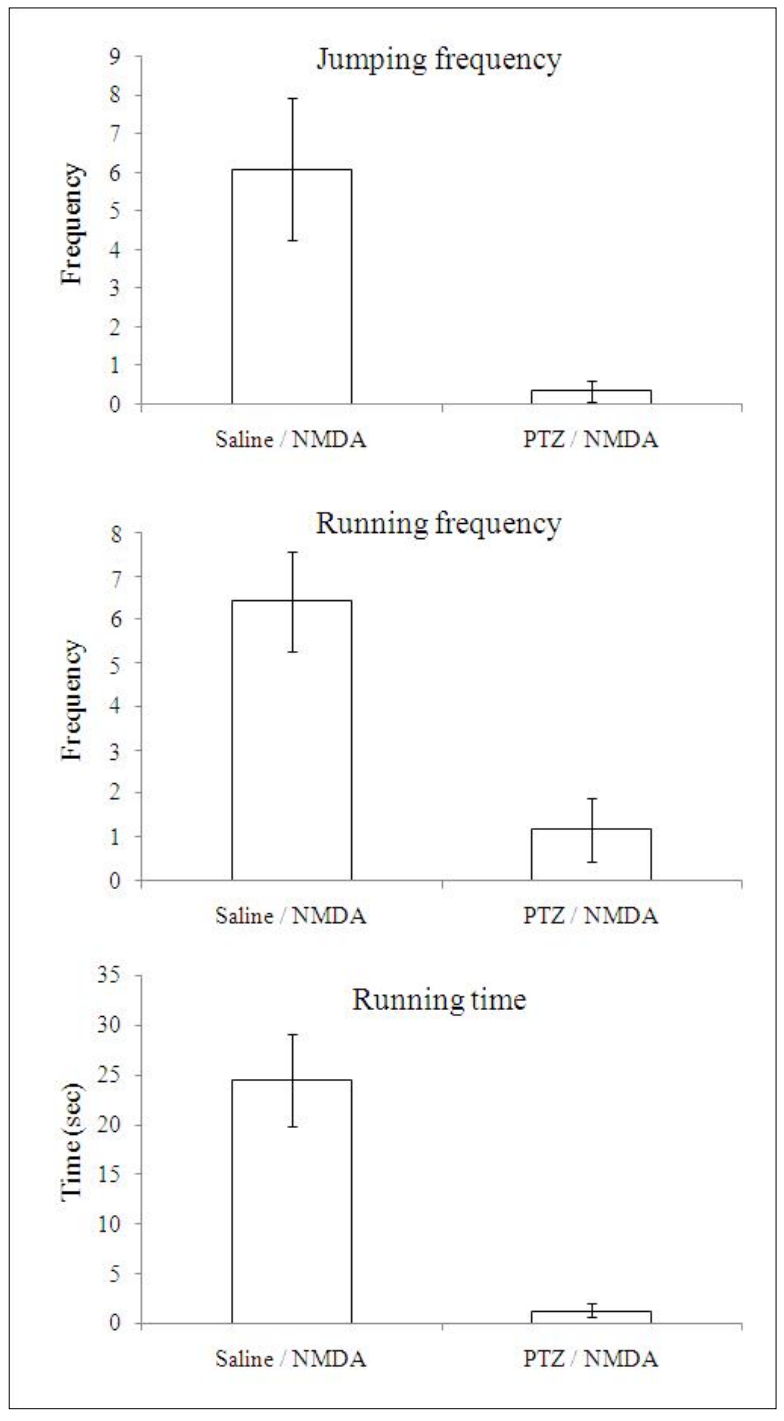

Figure 1 - Mean $( \pm$ SEM) jumping frequency (upper portion), running frequency (middle portion) and running time (lower portion $)$ induced by NMDA $(.3 \mu \mathrm{l}$ at $15.0 \mu \mathrm{g} / \mu \mathrm{l})$ microinjections into the DPAG among animals intraperitoneally injected with saline or PTZ $(15 \mathrm{mg} / \mathrm{kg})$.

by contextual fear conditioning) plays an inhibitory role in panic attack genesis (as modeled by NMDA stimulation of the DPAG).

Experiment II indicated that intraperitoneal PTZ injection was capable of inhibiting the escape responses induced by microinjection of NMDA into the DPAG. Although these results also corroborate the perspective that anxiety inhibits panic attacks, they are not in accordance with the report by Schenberg et al. (2001) showing that PTZ $(50 \mathrm{mg} /$ $\mathrm{kg}$ ) facilitated escape responses elicited by NMDA microinjection into the DPAG. Differences in the PTZ dose between Schenberg et al. (2001) and the present study could be responsible for these conflicting results. Doses from 10 to $20 \mathrm{mg} / \mathrm{kg}$ have been shown to be the prototypical anxiogenic doses utilized in animal models of anxiety (Ramos et al.,
2008), whereas doses ranging from 40 to $120 \mathrm{mg}$ / $\mathrm{kg}$ produce seizures (Velisek et al., 1992) and have been used as an animal model of epilepsy.

PTZ is a $\mathrm{GABA}_{\mathrm{A}}$ antagonist. DPAG has been shown to contain a significant number of GABAergic interneurons that play an important inhibitory role on the regulation of defensive behavior (Brandão et al. 1986; DiScala \& Sandner 1989). For example, DPAG microinjections of GABA receptor antagonists or inhibitors of glutamic acid decarboxylase, theenzymeresponsible forGABAsynthesis, produced unambiguous active defensive responses such as running and jumping (Brandão et al. 1982, 1986; Schmitt et al. 1985). Therefore, intraperitoneal injection of high doses of PTZ in the Shenberg et al. (2001) study might have led to a decrease in $\mathrm{GABA}_{\mathrm{A}}$ receptor function within the DPAG, thus facilitating escape reactions induced by stimulation of this area.

Several lines of evidence have suggested that anticipatory anxiety and panic attacks are mediated by distinct neural circuitries. Anxiety seems to be associated with limbic forebrain structures such as the amygdaloid complex, whereas panic attacks are related to more primitive hindbrain structures such as the DPAG. Fibers originating from the DPAG innervate various forebrain regions, including the amygdaloid complex, through the medial forebrain bundle (Cameron et at., 1995). Therefore, regular hyper-activation of the DPAG could lead to the development of PD through this ascending projection. On the other hand, activation of forebrain areas such as the amygdaloid complex produces several autonomic and behavioral reactions associated with anxiety through descending projections that reach hypothalamic nuclei and mesencephalic structures. Descending inhibitory projections might reach the DPAG, which could be responsible for the inhibitory role of anxiety in the occurrence of panic attacks, and this possibility is an important consideration for the interpretation of the present results.

\section{Acknowledgments}

A.P.M.C. and J.L.F. were supported by grants from the Brazilian National Research Council (CNPq, 350214/1998-0 and 522720/95-10). LFCP was supported by a FAPA grant from Universidad de los Andes. BOG and BCL were supported by graduate and undergraduate student fellowships from $\mathrm{CNPq}$, and $\mathrm{WJH}$ was an international student from the Netherlands.

\section{References}

Adler, C.M., Craske, M.G., Barlow, D.H. (1987). Relaxation-induced panic (RIP): When resting isn't peaceful. Integrative Psychiatry, 2, 94-112.

Bandler, R., \& Carrive, P. (1988). Integrated defense reaction elicited by excitatory amino acid microinjection in the midbrain periaqueductal grey region of the unrestrained cat. Brain Research, 439, 95-106. 
Basoglu, M., Marks, I.M., \& Sengun, S. (1992). A prospective study of panic and anxiety in agoraphobia with panic disorder. British Journal of Psychiatry, 160, 57-64.

Behbehani, M.M. (1995). Functional characteristics of the midbrain periaqueductal gray. Progress in Neurobiology, 46, 575-605.

Bittencourt, A.S., Carobrez, A.P., Zamprogno, L.P., Tufik, S., \& Schenberg, L.C. (2004). Organization of single components of defensive behaviors within distinct columns of periaqueductal gray matter of the rat: Role of N-Methyl-d-Aspartic Acid Glutamate Receptors. Neuroscience, 125, 71-89.

Bouton, M.E., Mineka, S., \& Barlow, D.H.A. (2001). A modern learning theory perspective on the etiology of panic disorder. Psychological Review, 108, 4-32.

Brandão, M.L., Aguiar, J.C., \& Graeff, F.G. (1982). GABA mediation of the antiaversive action of minor tranquilizers. Pharmacology, Biochemistry and Behavior, 16, 397-402.

Brandão, M.L., DiScala, G., Bouchet, M.J., \& Schmitt, P. (1986). Escape behavior produced by the blockade of glutamic acid decarboxilase GAD in mesencephalic central gray or medial hypothalamus. Pharmacology, Biochemistry and Behavior, 24, 497-502.

Brandão, M.L., Zanoveli, J.M., Ruiz Martinez, R.C., Oliveira, L.C., \& Landeira-Fernandez, J. (2008). Different patterns of freezing behavior organized in the periaqueductal gray of rats: association with different type of anxiety. Behavioral and Brain Research, 188, 1-13.

Cameron, A.A., Khan, I.A., Westlund, K.N., Cliffer, K.D., \& Willis, W.D. (1995). The efferent projections of the periaqueductal gray in the rat: a Phaseolus vulgaris-leucoagglutinin study. I. Ascending projections. Journal of Comparative Neurology, 351, 568-84.

Cassano, G.B., Perugi, G., \& McNair, D.M. (1988). Panic disorder: review of the empirical and rational basis of pharmacological treatment. Pharmacopsychiatry, 21, 157-65.

Cruz, A.P., Frei, F., \& Graeff, F.G. (1994). Ethopharmacological analysis of rat behavior on the elevated plus-maze. Pharmacology, Biochemistry and Behavior, 49, 171-6.

DiScala, G., \& Sandner, G. (1989). Conditioned place aversion produced by microinjections of semicarbazide into the periaqueductal gray of the rat. Brain Research, 483, 91-97.

DSM IV ${ }^{-T R}$ : (1995). Diagnostic and Statistical Manual of Mental Disorders. Porto Alegre: Artes Médicas.

Fanselow, M.S., Kim, J.J., Young, S.L., Calcagnetti, D.J., DeCola, J.P., Helmstetter, F.J., \& Landeira-Fernandez, J. (1991). Differential effects of selective opioid peptide antagonists on the acquisition of pavlovian fear conditioning. Peptides, 12, 1033-1037.

Gorman, J.M., Liebowitz, M.R., Fyer, A.J., Goetz, D., Campeas, R.B., Fyer, M.R., Davies, S.O. \& Klein, D.F. (1987). An open trial of fluoxetine in the treatment of panic attacks. Journal of Clinical Psychopharmacology, 7, 329-332.

Hettema, J.M. (2008). The nosologic relationship between generalized anxiety disorder and major depression. Depression and Anxiety, 2, 300-316.

Izumi T, Inoue T, Tsuchiya $\mathrm{K}$, Hashimoto $\mathrm{S}$, Ohmori T, Koyama $\mathrm{T}$. (1999).Effects of the benzodiazepine antagonist flumazenil on conditioned fear stress in rats. Progress in Neuro-Psychopharmacoly
\& Biological Psychiatry, 23, 1247-1258.

Kemp,A., \& Felmingham, K. (2008). The psychology and neuroscience of depression and anxiety: towards an integrative model of emotion disorders. Psychology \& Neuroscience, 2, 177-181.

Klein, D.F., \& Fink, M. (1962). Psychiatric reaction patterns to imipramine. American Journal of Psychiatry, 119, 432-438.

Klein, D.F., \& Klein, H.M. (1989). The definition and psychopharmacology of spontaneous panic and phobia. In: Psychopharmacology of Anxiety (pp. 135-162). Oxford: Oxford University Press.

Krieger, J.E., \& Graeff, F.G. (1985). Defensive behavior and hypertension induced by glutamate in the midbrain central gray of the rat. Brazilian Journal of Medical and Biological Research, 18, 61-67.

Landeira-Fernandez, J. (1996). Context and Pavlovian Conditioning. Brazilian Journal of Medical and Biological Research, 29, 149-173.

Liebowitz, M.R., Fyer, A.J., Gorman, J.M., Dillon, D., Appleby, I.L., Levy, G., Anderson, S., Levitt, M., Palij, M., \& Davies, S.O. (1984). Lactate provocation of panic attacks. I. Clinical and behavioral findings. Arquives of General Psychiatry, 41, 764-70.

Magierek, V., Ramos, P.L., da Silveira-Filho, N.G., \& Nogueira, R.L., \& Landeira-Fernandez, J. (2003). Context fear conditioning inhibits panic-like behavior elicited by electrical stimulation of dorsal periaqueductal gray. Neuroreport, 14, 1641-1644.

Molchanov, M.L., \& Guimarães, F.S. (1999). Defense reaction induced by a metabotropic glutamate receptor agonist microinjected into the dorsal periaqueductal gray of rats. Brazilian Journal of Medical and Biological Research, 32, 1533-1537.

Nashold Jr., B.S., Wilson, W.P., \& Slaughter, D.G. (1969). Sensations evoked by stimulation in the midbrain of man. Journal of Neurosurgery, 30, 14-24.

Paxinos, G. \& Watson, C. (1986). The rat brain in stereotaxic coordinates, Academic Press, San Diego.

Ramos, A., Pereira, E., Martins, G.C., Wehrmeister, T.D., \& Izídio, G.S. (2008). Integrating the open field, elevated plus maze and light/dark box to assess different types of emotional behaviors in one single trial. Behavioral and Brain Research, 193, 277-288.

Schenberg, L.C., Bittencourt, A.S., Sudré, E.C., \& Vargas, L.C. (2001). Modeling panic attacks. Neuroscience Biobehavioral Review, 25, 647-659.

Schmitt, P., Di Scala, G., Brandão, M.L., \& Karli, P. (1985). Behavioral effects of microinjections of SR 95103, a new GABA-A antagonist, into the medial hypothalamus or the mesencephalic central gray. European Journal of Pharmacology, 11, 149-158.

Targum, S.D., \& Marshall, L.E. (1989). Fenfluramine provocation of anxiety in patients with panic disorder. Psychiatry Research, 28 295-306

Vargas, L.C., \& Schenberg, L.C. (2001). Long-term effects of clomipramine and fluoxetine on dorsal periaqueductal grey-evoked innate defensive behaviours of the rat. Psychopharmacology (Berl), $155,260-268$

Velisek, L., Kubova, H., Pohl, M., Stankova, L., Mares, P., \& Schickerova, R. (1992). Pentylenetetrazol-induced seizures in rats: an ontogenetic study. Naunyn Schmiedebergs Archives of Pharmacology, 346, 588-591. 